\title{
Development of an electrospinning method for the tensile testing of single nanofibers
}

\author{
K. Tanaka ${ }^{1}$, Y. Minamiguchi ${ }^{2}$, Y. Miyoshi ${ }^{2}$, \\ T. Katayama ${ }^{1} \&$ S.-H. Hyon ${ }^{3}$ \\ ${ }^{1}$ Department of Biomedical Engineering, Doshisha University, Japan \\ ${ }^{2}$ Department of Mechanical Engineering, Doshisha University, Japan \\ ${ }^{3}$ Institute for Frontier Medical Sciences, Kyoto University, Japan
}

\begin{abstract}
Nanofibers are expected to be widely used in many applications, such as filters, catalyst supports and scaffolds. The electrospinning process is an easy method by which to produce nanofibers from polymer solutions or melts. For the use of electrospun nanofibers, the mechanical characterization of single nanofibers should be investigated. However, few researches for the mechanical testing of a single nanofiber have been conducted. The mechanical characterization of a single nanofiber is difficult because of the difficultly of measuring the small load and handling a single nanofiber. To overcome these issues, it is necessary to spin single nanofibers for the tensile testing of single nanofibers.

Poly(lactic acid)(PLLA), whose melting point is about $180^{\circ} \mathrm{C}$, has high mechanical properties in biodegradable plastics. However, its melting point is not high enough in some applications. A stereocomplex-type PLA(SCPLA) blending of PLLA and PDLA has a melting point of $230^{\circ} \mathrm{C}$, which is about $50^{\circ} \mathrm{C}$ higher than that of PLLA.

In this study, an electrospinning method for the tensile testing of single nanofibers is developed using the electrical potentials difference between two $\mathrm{Cu}$ strips of target. The tensile testing of a single nanofiber was successfully conducted using a nanoscale tensile testing machine. The tensile strength of a single SCPLA nanofiber fabricated by the electrospinning was $223 \pm 75 \mathrm{MPa}$.
\end{abstract}

Keywords: electrospinning, nanofiber, poly L-lactic acid (PLA), tensile test, stereocomplex-type PLA. 


\section{Introduction}

Due to the high surface area to volume ratio, nanofibers are expected to be used for a wide range of applications, such as tissue engineering, filter media and reinforcements in composites $[1,2]$. The electrospinning technique is a simple method for the production of nanofibers. In this electrospinning technique, to blow out a polymer solution from a needle hole, an electric field is applied between a polymer solution and a target. During the electrospinning process, the solvent evaporates and polymer nanofibers are fabricated on the target [3-5].

Poly L-lactic acid (PLLA), which can be produced by corns, sugars or beets, is a thermoplastic resin with a melting temperature of about 180 degrees Celsius. Although this melting temperature is fairly high compared to the other biodegradable polymers, its melting temperature is not high enough in some applications. An equimolar mixture of poly L-lactide (PLLA) and poly D-lactide (PDLA) can be crystallized into a stereocomplex-type PLA (SCPLA). The melting point of stereocomplex crystal is 50 degrees Celsius higher than that of PLLA [6]. Since these nanofibers are often subjected to stress and strains, their mechanical properties should be investigated. Due to the difficulty in handling individual nanofibers, few researches for mechanical testing of single nanofibers have been conducted [7]. The standard method of tensile test for a single nanofiber has not been established yet.

In this study, electrospinning method for tensile test of single nanofibers is developed using the electrical potentials difference between two $\mathrm{Cu}$ strips of target. The tensile test was conducted to characterize mechanical properties of single electrospun SCPLA nanofibers.

\section{Electrospinning method}

\subsection{Electrospinning of stereocomplex-type PLA}

Stereocomplex-type PLA blended by PLLA/PDLA was used in this study. The PLLA (weight-average molecular-weight, $\mathrm{Mw}=2.7 \times 10^{5} \mathrm{~g} / \mathrm{mol}$ ) and PDLA (Mw $\left.=1.8 \times 10^{5} \mathrm{~g} / \mathrm{mol}\right)($ PLLA $/$ PDLA $=1: 1)$ were blended. Two kinds of solvents were mixed at different concentration. DCM (DCM: dichloromethane) is used for solvent purpose. DMF (DMF: dimethylformamide) was added to control solution conductivity. It is known that higher solution conductivity results in the formation of nanofibers with less beads and smaller diameters. DMF has a dielectric constant of 37, whereas DCM has a dielectric constant of 89. Polymer was first dissolved in DCM before DMF was added. The nanofibers were fabricated from $8 \mathrm{wt} \%$ polymer solution with DCM and DMF in the ratio of 8:2 $(\mathrm{w} / \mathrm{w})$ as the solvent. Nanofibers were fabricated using Nanofiber Electrospinning Unit (NEU, Kato Tech) shown in Fig.1. Electrospinning was conducted at room temperature $\left(18 \pm 3^{\circ} \mathrm{C}\right)$, with relative humidity at $40-60 \%$. An applied voltage was set for $15 \mathrm{kV}$ between the needle for polymer solution and the target with a flow rate of polymer solution of $3.0 \times 10^{-4} \mathrm{ml} / \mathrm{sec}$. 


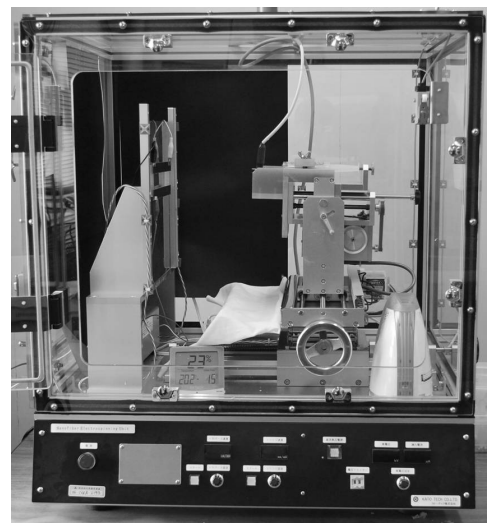

Figure 1: Nanofiber electrospinning unit.

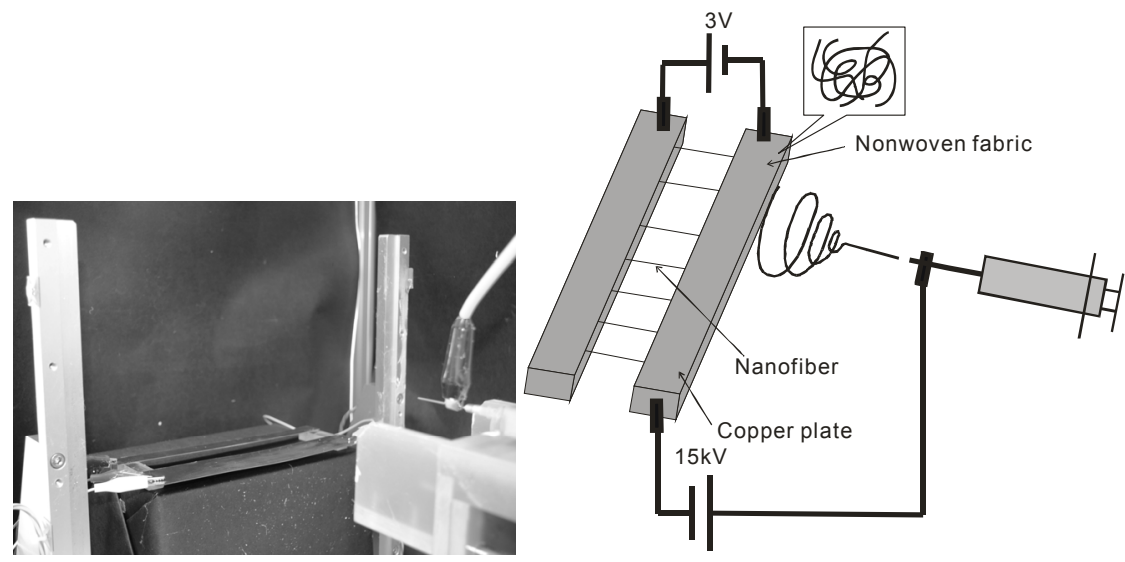

Figure 2: Device for fabrication of Figure 3: Schematic drawing of single nanofibers. fabrication of a single nanofiber.

\subsection{Electrospinning target of single nanofibers}

Two $\mathrm{Cu}$ strips which were placed parallel with $30 \mathrm{~mm}$ distance were used for target as shown in Fig.2. Circuit and electrodes were placed as shown in Fig.3. The electrical potentials difference between two $\mathrm{Cu}$ strips was set at $3 \mathrm{~V}$.

\subsection{Results and discussion}

Fig.4 shows the optical microscope image of the electrospun fibres. The few aligned fibres were successfully spun perpendicularly within two parallel $\mathrm{Cu}$ strips. Fig.5 shows the SEM image of the electrospun fibres which was spun on 


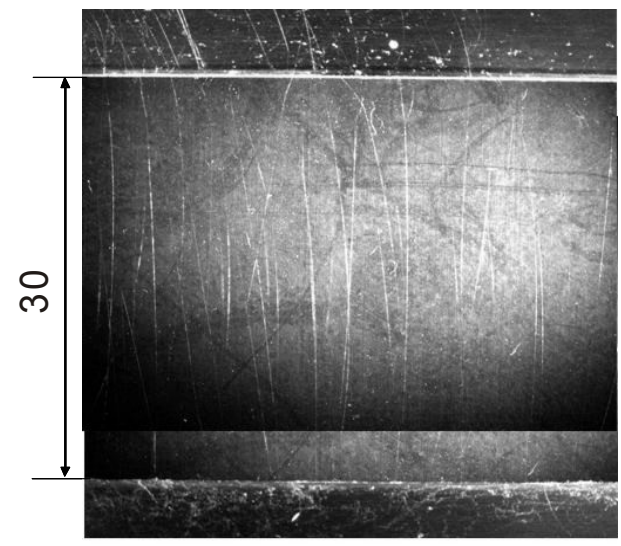

Figure 4: Electrospun nanofibers Figure 5: between two $\mathrm{Cu}$ strips.

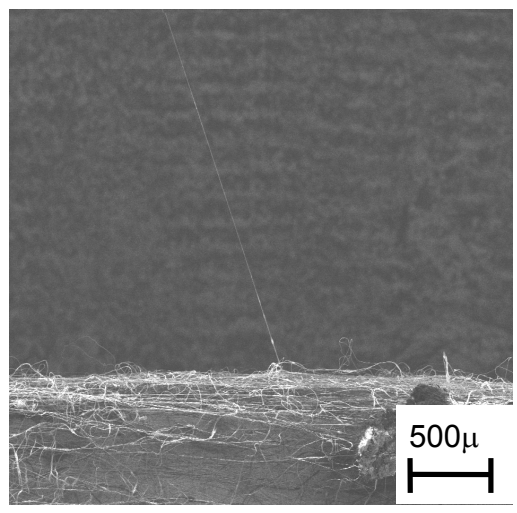

SEM image of the border of the front a $\mathrm{Cu}$ strip.
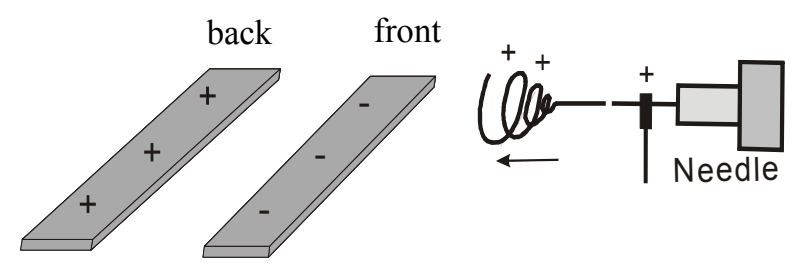

(a)

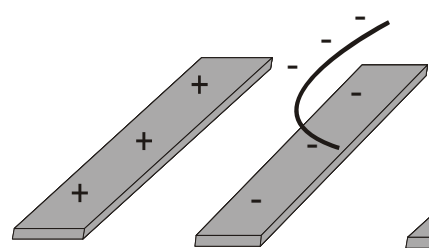

(b)

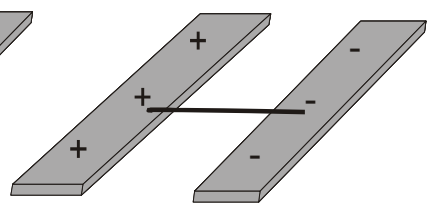

(c)

Figure 6: Mechanism of fabrication of a nanofiber.

the front $\mathrm{Cu}$ strip. A single nanofiber is derived from the nonwoven fabric. Most of the nanofibers were spun on the front $\mathrm{Cu}$ strip. Only a portion of the spun fibres get spun in between the two $\mathrm{Cu}$ strips. Fig.6 shows the electrospinning process for fabrication of a single nanofiber. When the positively charged polymer jet reached the negatively charged front $\mathrm{Cu}$ strip (Fig.6a $\rightarrow$ b), the remaining part of the nanofiber was negatively charged. After that, negatively charged jet will be pulled towards the positively-charged back $\mathrm{Cu}$ strip $($ Fig.6b $\rightarrow c)$. 


\section{Tensile test of single electrospun nanofibers}

\subsection{Specimen preparation and test conditions}

Specimens for tensile test were prepared by using aligned nanofibers (as mentioned in section 2.3). Tensile test specimens were prepared as following step (Fig.7).

(1) Two Al tabs were bonded to a paper tab by double sided tapes.

(2) Conductive tapes were put on Al tabs.

(3) Collect a nanofiber between the two $\mathrm{Cu}$ strips onto the conductive tape.

(4) The single fibre was bonded by adhesive.

The tensile test of as-spun nanofiber was conducted using a nano tensile testing machine (NanoTensile, Hysitron). At a displacement rate of $10 \mu \mathrm{m} / \mathrm{s}(0.6$ $\mathrm{mm} / \mathrm{min}$ ), this testing machine has $500 \mathrm{mN}$ load range and $12.5 \mathrm{nN}$ load resolution.

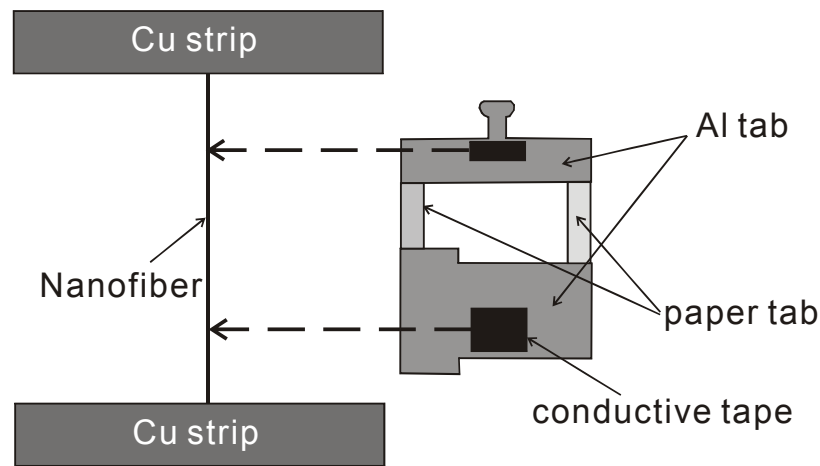

Figure 7: $\quad$ Procedure of specimen preparation.

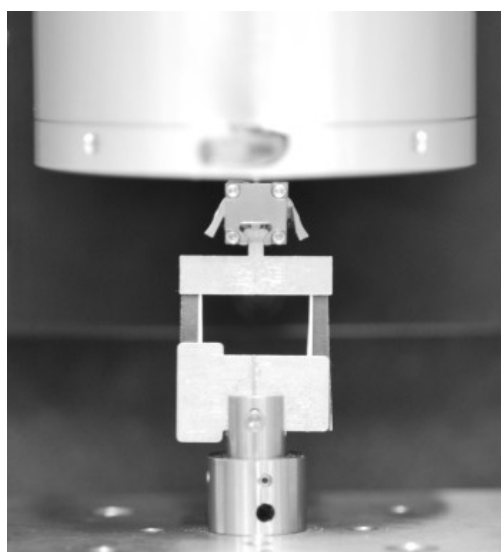

Figure 8: $\quad$ Specimen fixed on Nanotensile $^{\circledR}$. 
NanoTensile is a nanoscale tensile testing machine equipped with the $\mathrm{X} / \mathrm{Y}$ staging system. The $\mathrm{X} / \mathrm{Y}$ staging system is used to perform an automatic alignment of the specimen before the tensile test. Therefore, this testing machine is suitable for the precise tensile test of nanofibers. Fig. 8 shows a specimen fixed on the tensile testing machine.

Diameters of nanofibers were measured using a scanning electron microscope (JSM-6390LT, JEOL). After tensile test, the diameter of nanofiber which bonded to conductive tape was measured. Average of diameter at five points was used to calculate the tensile strength.

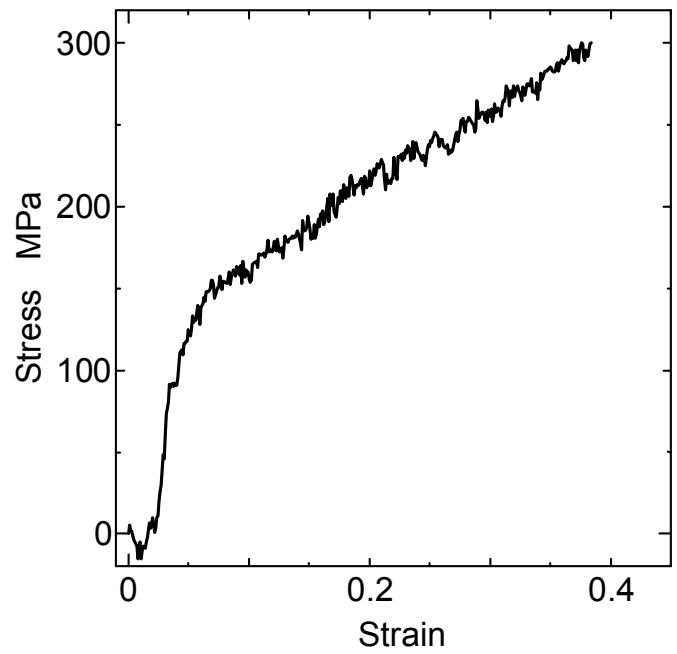

Figure 9: Stress-strain curve of a single electrospun nanofiber.

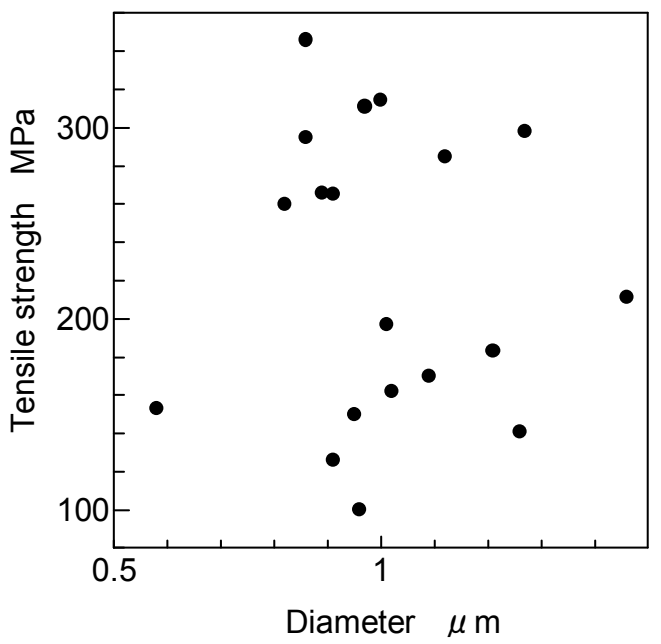

Figure 10: Relationship between tensile strength and diameter. 


\subsection{Results and discussion}

The measured diameter of electrospun nanofibers for the tensile test was $1.0 \pm$ $0.2 \mu \mathrm{m}$.

Typical stress-strain curves of single electrospun nanofibers are shown in Fig.9. All results of tensile test are shown in Fig.10. Tensile strength was $223 \pm$ $75 \mathrm{MPa}$. For PLLA nanofibers, tensile strength was reported as $204 \pm 86 \mathrm{MPa}$ [8], which is close to our result. The stress-strain curve in Fig.9 consists of two linear stages, which is similar to that of the case of polyimide nanofibers [9]. After elastic deformation, large plastic deformation was observed.

\section{Discussion of the developed electrospinning method}

In the previous study for tensile test of single nanofibers, the single nanofibers were pulled out from nonwoven fabric by a glass stick [10]. However, there was the possibility that the fibre could be suffered plastic deformation when specimen was prepared by this method. Therefore, the load when being pulled out from nonwoven fabric by the glass stick is necessary to be measured.

The specimens for this pick-up test have been prepared by the same method shown in chapter 2-2. As shown in Fig.5, the nonwoven fabric was spun on two $\mathrm{Cu}$ strips and the single nanofiber was derived from the nonwoven fabric; the load of the single nanofiber being pulled out from the nonwoven fabric by the glass stick can be measured by this pick-up test. Nanotensile 5000 shown in chapter 3-1 was used for pick-up test at the tensile speed of $10 \mu \mathrm{m} / \mathrm{s}$. Five specimens were used for pick-up test. The overview of this pick-up test is shown in Fig. 11.

The load-displacement curve of the pick-up test was shown in Fig.12. At sharp drops of load in the load-displacement curve, picked-up fibre may be detached from other fibres or $\mathrm{Cu}$ strips. The maximum load of the loaddisplacement curve of the pick-up test was $0.54 \pm 0.22 \mathrm{mN}$. As shown in chapter $3-2$, the average diameter of nanofibers in this study was $1 \mu \mathrm{m}$. When the fibre

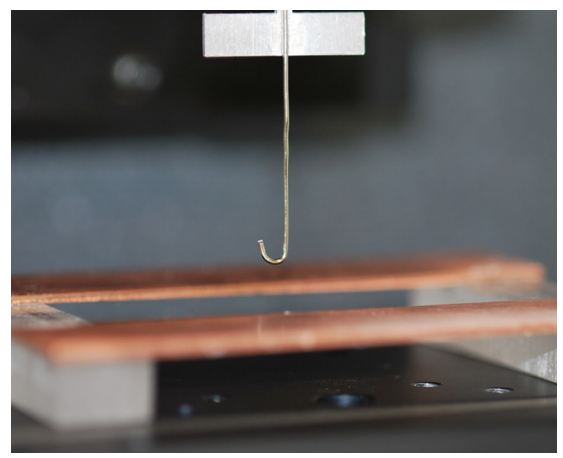

Figure 11: Pick-up test. 


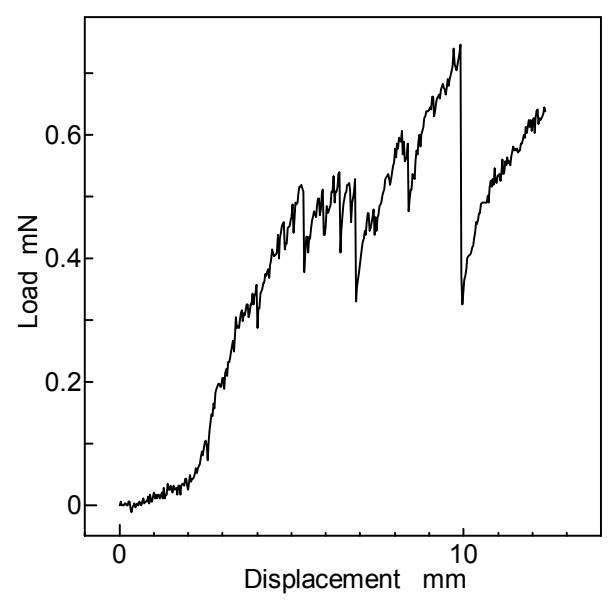

Figure 12: Load-displacement curve of the pick-up test.

diameter was assumed to be $1 \mu \mathrm{m}$, the maximum calculated stress applied to fibres during pick-up test was $164 \pm 26 \mathrm{MPa}$. This stress exceeded the elastic region by comparison within the stress-strain curve shown in Fig.9, so the fibre may be suffered by plastic deformed during specimen preparation. Therefore, to prepare specimen for tensile test of single nanofibers, the developed electrospinning method with electrical potential difference between two $\mathrm{Cu}$ strips of target is suitable.

\section{Conclusions}

Electrospinning method for tensile test of single nanofibers is developed using the electrical potentials difference between two $\mathrm{Cu}$ strips of target. The tensile test was conducted to characterize mechanical properties of single electrospun SCPLA nanofibers. The investigation yielded the following conclusions.

1. Electrospinning method using the electrical potentials difference between two $\mathrm{Cu}$ strips of target for single tensile test of nanofibers has been developed.

2. The tensile strength of a single SCPLA nanofiber fabricated by the electrospinning was $223 \pm 75 \mathrm{MPa}$.

\section{Acknowledgement}

This research was partially supported by the Ministry of Education, Culture, Sports, Science and Technology; Grant-in-Aid for Young Scientists (B), 19760079, 2008. 


\section{References}

[1] D. Li, M. W. Frey, A. J. Baeumner, Electrospun polylactic acid nanofiber membranes as substrates for biosensor assemblies, Journal of Membrane Science, 279, pp.354-363, 2006.

[2] R. S. Ruoff, Special issue on nanocomposites, Composites Science and Technology, 66, pp.1099-1101, 2006.

[3] Y Ishii, H Sakai, H Murata, A new electrospinning method to control the number and a diameter of uniaxially aligned polymer fibers, Materials Letters, 62, pp.3370-3372, 2008.

[4] S. Ramakrishna, K. Fujiwara, W-E. Teo, T-C. Lim, Z. Ma, An Introduction to Electrospinning and Nanofibers, World Scientific Publishing Co. Pte. Ltd, pp.1-21, 2005

[5] R. Inai, M. Kotaki, S. Ramakrishna, Structure and properties of electrospun PLLA single nanofibers, Nanotechnology, 16, pp.208-213, 2005.

[6] Y. Furuhashi, Y. Kimura, N. Yoshie, H. Yamane, High-order structures and mechanical properties of stereocomplex-type poly(lactic acid) melt spun fibers, Polymer, 47, pp.5965-5942, 2006.

[7] H. Tsuji, M. Nakano, M. Hashimoto, K. Takashima, S. Katsura, A. Mizuno, Electrospinning of Poly(lactic acid) Stereocomplex Nanofibers, Biomacromolecules, 7, pp.3316-3320, 2006.

[8] E. P. S. Tan, C. T. Lim, Effects of annealing on the structural and mechanical properties of electrospun polymeric nanofibres, Nanotechnology, 14, pp.2649-26547, 2006.

[9] F. Chen, X. Peng, T. Li, S. Chen, X. Wu, D. H. Reneker, H. Hou, Mechanical characterization of single high-strength electrospun polyimide nanofibers, J. Phy. D: Appl. Phys, 41, pp.1-8, 2008

[10] K Tanaka, Y Miyosi, T Katayama and Suong-HyuHyon, Evaluation of Mechanical Properties of a Single Electrospun SCPLA Nanofiber, IWGC-5, pp.13-16, 2008. 